\title{
AIRBORNE AND MOBILE LIDAR, WHICH SENSORS FOR WHICH APPLICATION?
}

\author{
J. Vallet ${ }^{1}$, A. Gressin ${ }^{2}$, P. Clausen ${ }^{3}$, J. Skaloud ${ }^{3}$ \\ ${ }^{1}$ Helimap System SA, Epalinges, Switzerland - julien.vallet@helimap.ch \\ ${ }^{2}$ University of Applied Sciences Western Switzerland (HES-SO / HEIG-VD), Insit Institute - adrien.gressin@heig-vd.ch \\ ${ }^{3}$ Geodetic Engineering Laboratory (TOPO), Swiss Federal Instutute of Technology (EPFL), Lausanne, Switzerland - \\ (philipp.clausen,jan.skaloud)@epfl.ch
}

\section{TC I, Sensor Systems}

\section{KEY WORDS: LiDAR, UAV, Automotive, IMU, MEMS}

\begin{abstract}
The UAV mapping industry expanded tremendously during the last five years. Thanks to miniaturization, automation and advertising, this technology may give a wrong impression that mapping of certain quality is as simple as clicking few buttons on a PC. Moreover, with a large and continuously increasing offer of hardware and software, the identification of the right tools is not easy, especially when aiming at certain standard. In this respect, the mapping with LiDAR is more delicate than with a camera due to a lower level of redundancy within the process of orientation/georeferencing and somewhat higher threshold on the size/weight per performance ratio within these sensors. This fact motivated us to present a practical benchmark evaluating a popular small LiDAR sensor in realistic conditions for intrinsic parameters such as noise or capacity to penetrate canopy, as well as the "low-weight" inertial technology in terms of geometrical influences on the resulting point cloud. The practical limitations are indeed considerably lower than those specified by the manufacturers or tested in laboratory conditions. These should be considered together with other "mapping-productivity" factors that are summarized in the last part of this study.
\end{abstract}

\section{INTRODUCTION}

It is over half a decade since UAV mapping invaded the world of geomatics, promising to solve almost any mapping challenges at a low cost. Business expanded drastically (Colomina and Molina, 2014), sometimes faster than flight regulations, often based on promises of numerous emerging drone manufacturers and new generations of drone mapping software surfing on the UAV "wave". Sensor miniaturization supported the rise of the UAV industry, with the availability of smaller and lighter devices. Since about two years, it is not uncommon to see advertisements for achieving sub-centimeter accuracy with either photogrammetric or LiDAR equipped UAV's without additional commodities according to the catchphrase "What you click is what you map (WYCWYM)".

Indeed, the process of data acquisition and production appear really easy with drones thanks to intuitive flight planning, reliable drone guidance, and subsequent automation of data processing. Nevertheless, it is the experience of the operator in the setup and evaluation that designs sufficient redundancy and thus controllability that guards against unsuitable or erroneous deliverables. This process, however, remains quite challenging in LiDAR-based products, where the quality of distance observations as well as georeferencing produced by lightweight sensors may vary rapidly in time and space.

This fact motivates us to present a practical benchmark that sketches a realistic portrait of small airborne/mobile LiDAR and georeferencing sensors in terms of orientation and mapping performance. After introducing its design, we present a detailed comparison of sensors in terms of realistic accuracy as well as practical aspects related to their deployment and "mapping productivity".

\section{EXPERIMENTAL SETUP}

\subsection{Equipment}

\subsubsection{Airborne sensors}

The aim of the experimental setup is to compare different sensors with respect to a reference during the same flight and thus acquisition conditions. Indeed, comparing sensors in different flights modifies the un-mastered parameters such as GNSS constellation or certain aspects of flight dynamic, fact of which may bias the interpretation. The tested equipment comprises of a reference and UAV-grade LiDARs that are embarked on the same airborne platform together with a reference camera, small "UAV-type" cameras, as well as a reference and small inertial measurement units. The system is flown by helicopter over an area at a speed that is typical for small UAV's (multi-copter) to provide the same conditions for all sensors in terms of temperature, dynamics and height $\mathrm{AGL}^{1}$. All sensors are rigidly mounted to the same assembly that is vibration dampened.

Three different IMU's are used:

- $\quad$ Reference, navigation-grade INS (AIRINS, Ixblue)

- UAV-grade MEMS-IMU (APX15, Trimble-Applanix)

- 4 low-cost MEMS-IMU's (NavChip v1/2011, Thales) mounted on a modified Gecko board (Kutler, 2012).

The GNSS signal provided by an airborne-grade dual frequency antenna is split between Javad (Delta TRE-3) and APX15 (Trimble) receivers, the former providing the time scale (1PPS) to AIRINS, NavChip IMUs as well to two LiDAR devices:

- $\quad$ Reference, medium-range VQ480 (Riegl)

- An automotive LiDAR PUCKLite (Velodyne)

Two cameras, an IXAR180 (PhaseOne) with 80 megapixels and $42 \mathrm{~mm}$ lens, and an A6000 (Sony) with $20 \mathrm{~mm}$ lens and 24 megapixels, complete the assembly depicted in Figure 1.

\footnotetext{
${ }^{1}$ Above Ground Level
} 


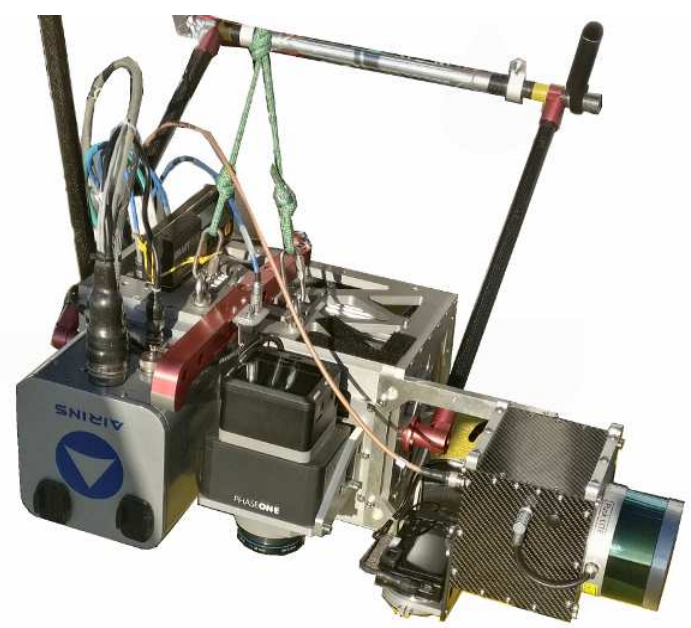

Figure 1: Assembly of 3 IMU's, 2 LiDARs and 2 cameras.

\subsubsection{Mobile mapping sensors}

In a similar way to the airborne assembly, the very same PUCKLite (Velodyne) and APX15 (Trimble/Applanix) sensors were placed on a car together with a reference unit: dual head VUX1-HA (Riegl) LiDAR and the navigation grade IMU (IGIIIe). Due to the configuration of the reference unit installed on the back of the car, the tested sensors needed to be placed on its front cowl using car chassis as a "semi-rigid" connection between them (Figure 2). The sensors were driven through a semi-urban area.
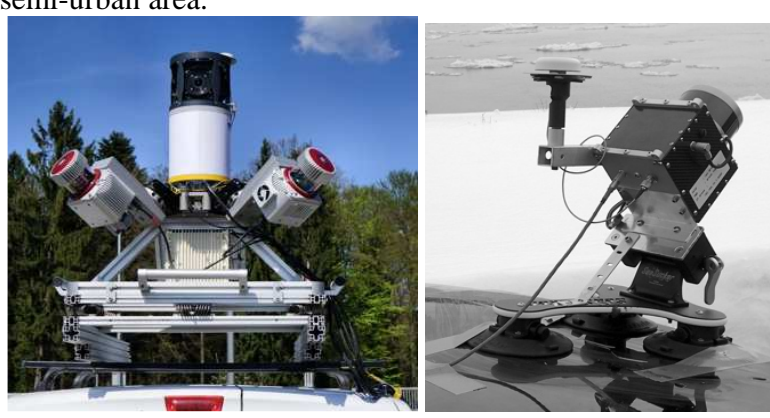

Figure 2: Rieg1/IGI sensors (back) and PuckLite/APX15 (front)

\subsection{Experimental sites}

\subsubsection{Airborne Test Area}

The chosen area has a surface of 60 ha $(300 \mathrm{~m} \mathrm{x} 2 \mathrm{~km})$ and features different terrain types including urban and rural areas, forest, agriculture fields, road, railroad and power lines. Twelve GCP's were distributed over the zone and their coordinates measured by static GNSS survey with an accuracy better than 2 $\mathrm{cm}$ (Figure 3).

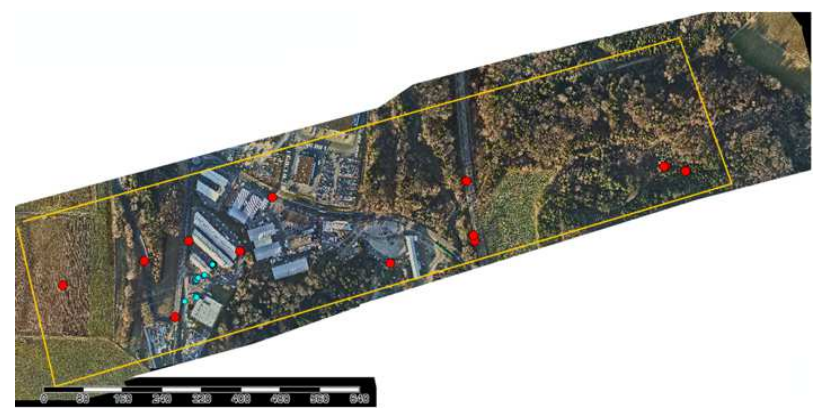

Figure 3: Experimental site near Aclens, Switzerland. Red dots represent GCPs and blue dots the check points.
Additional check points have been deployed on some surfaces and measured by RTK GNSS over a short base. To calibrate the small optical sensors in terms of boresight and interior orientation parameters prior the test zone, a second, smaller urban area has been used within the same flight. This contained houses with roofs of different slope and orientation as well as a set of 10 GCP's. This area was flown over in clover leaf pattern as shown in the upper left part of Figure 4.

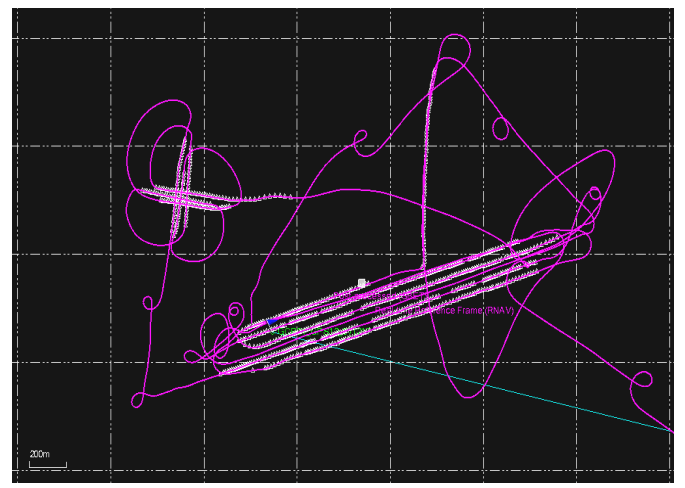

Figure 4: Trajectory of the flight. The clover leaf pattern in the NW represents the calibration flight. The $360^{\circ}$ turns are performed after certain time to limit the drift in heading.

\subsection{Airborne Data Acquisition}

To ensure the same observation conditions as well as the compliance with flight regulation and safety over urban and high voltage power lines the flight was conducted with a helicopter and not with a UAV. The flight was performed during a winter period (Dec. 2017) at 2 different heights above ground level to comply with range limitation of each scanner and to prevent interferences between the respective laser beams. The scanner and flight parameters are summarized in Table 1.

\begin{tabular}{cccccccc}
\hline $\begin{array}{l}\text { LiDAR } \\
\text { sensor }\end{array}$ & $\begin{array}{c}\text { Height } \\
\text { AGL } \\
{[\mathrm{m}]}\end{array}$ & $\begin{array}{c}\text { GSD } \\
{[\mathrm{cm}]}\end{array}$ & $\begin{array}{c}\text { TOF } \\
{[\mathrm{min}]}\end{array}$ & $\begin{array}{c}\# \\
\mathrm{img}\end{array}$ & $\begin{array}{c}\text { Speed } \\
{[\mathrm{km} / \mathrm{h}]}\end{array}$ & $\begin{array}{c}\text { Nominal } \\
\text { Density } \\
{\left[\mathrm{pt} / \mathrm{m}^{2}\right]}\end{array}$ & $\begin{array}{c}\text { Overlap } \\
\text { Lon/side } \\
{[\%]}\end{array}$ \\
\hline \hline VQ480 & 230 & 3.7 & 10 & 50 & 50 & 70 & $75 / 35$ \\
PUCK & 50 & 1.8 & 20 & 200 & 12 & $90-100$ & $75 / 35$ \\
\hline LiDAR & $\begin{array}{c}\text { Beam } \\
\text { divergence } \\
\text { sensor }\end{array}$ & $\begin{array}{c}\text { Point meas. } \\
\text { Rate }\end{array}$ & $\begin{array}{c}\text { Scan rate } \\
{[\mathrm{mrad}]}\end{array}$ & $\begin{array}{c}\text { Return } \\
\text { mode }\end{array}$ \\
\hline \hline VQ480 & 0.27 & & 200 & 100 & multi \\
PUCK & 3 & 300 & $10 x 16$ beams & dual \\
\hline
\end{tabular}

Table 1: Flight and acquisition parameters for both LiDAR configurations (TOF - flight duration over zone)

\subsection{Mobile Data Acquisition}

The mobile data acquisition was performed in the northern part of Lausanne ${ }^{2}$, Switzerland, where the road environment is composed of residential area, a 3 lanes road with 2 bridges and a parking lot (Figure 5). The area allows varying the observation distances between the car and its environment. The dual sensor head setup of Riegl-IGI generates a point density of $1^{\prime} 500 \mathrm{pt} / \mathrm{m}^{2}$ for each scanner at $50 \mathrm{~km} / \mathrm{h}$ on pavement, whereas PUCK provides $\sim 1000 \mathrm{pt} / \mathrm{m}^{2}$ at $18 \mathrm{~km} / \mathrm{h}$ and $500 \mathrm{pt} / \mathrm{m}^{2}$ at 36 $\mathrm{km} / \mathrm{h}$, respectively.

\footnotetext{
${ }^{2}$ Between Helimap office in Epalinges and Chalet-à-Gobet.
} 


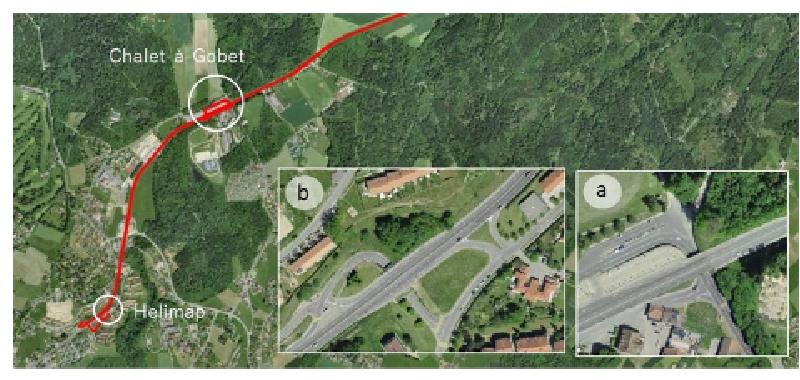

Figure 5: Trajectory for testing Puck-APX15 mobile mapping devices with respect to a reference (Riegl-IGI).

\section{COMPARISON METHOD}

The fact of having all sensors installed on the same assembly allows comparing them under the same flight/drive conditions (i.e., dynamic, GNSS constellation, and atmosphere). The evaluation focuses on three components, namely:

- The quality of the trajectory (mainly the attitude) and its effect on the absolute accuracy of the point cloud

- The level of noise in the point cloud

- The capacity to detect small object and the ability to penetrate the high vegetation

As photogrammetry is also an airborne mapping technique, we compare over the same (open) area the point clouds derived from UAV-LiDAR and UAV-photogrammetry (performed the same day with a high-end Sony camera RX1RII). We present more details about UAV photogrammetric point clouds quality in a separate contribution (Gressin et al, 2020).

\subsection{Software}

Trajectories were computed using the following software: APPS (IXBLUE) for integrating AIRINS data in loosely coupled manner with PPK $^{3}$ obtained via Novatel's GrafNAV; POSPAC (Trimble/Applanix) for tightly coupled integration of APX15 observations, Posproc 2.0 (Applanix) with internally designed filter for loosely integrating NavChip (either raw, pre-calibrated or redundant) observations with PPK.

The registration of point cloud was performed with the Riprocess suite (Riegl) and LIEO (Skaloud, 2017). Direct georeferencing of imagery was computed from INS/GNSS trajectory with CAMEO (Skaloud and Legat 2006).

\subsection{Orientation aspects}

Direct georeferencing (DG) or direct sensor orientation (DiSO), is a mandatory component of mobile LiDAR systems that has an important influence on the accuracy of the point cloud (Glennie, 2007). The error budget of a calibrated system with high-quality IMU flying at low altitude above the terrain is dominated by the positioning component, while the influence of attitude errors rises with the flying height and lower quality inertial sensors (Baltsavias, 1999, Schenk, 2001, Glennie 2007). Irrespectively of IMU quality due to observability reason, the attitude error in heading is generally 2-3 larger than in roll and pitch.

The attitude quality between small IMU types can be evaluated using the navigation grade (the most accurate) system as reference. Indeed, AIRINS has one of the most accurate fibro optic gyros used within the airborne mapping market. The APX15 has small temperature-calibrated sensors that are aimed

${ }^{3}$ Post Processed Kinematic $=$ carrier-phase differential GNSS to professional UAVs. Table 2 shows the manufacturer specification of each tested IMU. The NavChip sensors are according to manufacture specifications of a lower quality than those of APX15. However, as the Gecko4Nav board comprises of 4 NavChip MEMS IMU, these can be used in a redundant way. For that we adopt the method of synthetic low cost IMU's described in (Clausen and Skaloud, 2020) that involves switchon bias determination, with the goal to reach - in terms of noise level - a comparable performance to Applanix APX15 while having smaller systematic effects. Indeed, this fusion technique seems to be promising in reducing the cost of embarked IMU's on close-range sensors or inertial photogrammetry while shortening the duration of the in-flight calibration procedure.

\begin{tabular}{ccccc}
\hline & $\begin{array}{c}\text { Gyro drift } \\
{[\% / \mathrm{h}]}\end{array}$ & $\begin{array}{c}\text { Acc. Drift } \\
{[\mathrm{mg}]}\end{array}$ & $\begin{array}{c}\text { Accuracy PPK } \\
\text { RP/H }\left[{ }^{\circ}\right]\end{array}$ & $\begin{array}{c}\text { Accuracy PPK } \\
\text { XYZ }[\mathrm{m}]\end{array}$ \\
\hline \hline AIRINS & 0.01 & $<0.5$ & $0.002 / 0.005$ & 0.03 \\
APX15 & 10 & 2 & $0.08 / 0.03$ & 0.03 \\
NavChipV1 & 10 in-run & -- & -- & -- \\
\hline
\end{tabular}

Table 2: IMU manufacturer specifications

The pre-requisite for direct comparison of attitude among the systems is the removal of the respective orientation-installation offsets, so called boresight. These values can be, for-instance, obtained from camera-imu boresight calibration.

\subsection{Point cloud comparison}

Even more interesting is, to compare point clouds based on the very same LiDAR but georeferenced with different inertial technologies. However, this can only be performed on the airborne dataset as the respective LiDAR units mounted on the car are not rigidly connected. Thus, it is possible to only compare PUCK-APX15 point cloud with the one generated via Riegl-IGI tandem. The GCP and check points enable evaluation of the absolute altimetric accuracy of the point cloud.

\subsubsection{Noise aspects}

The use of automotive low-cost LiDARs for mapping is on one side interesting due to its price, but challenging on the other hand, because these sensors were designed for different application. If the range's noise for mapping LiDAR is about 1$2 \mathrm{~cm}$ even at long distances $(>500 \mathrm{~m})$, the automotive sensor based on solid state LiDAR exhibit a noise level of 3-4 $\mathrm{cm}$ as assessed in laboratory conditions with rigid targets (Glennie et al., 2016). Hence, the characterization of noise level on various mapping surfaces is novel and relevant information.

A practical technique to analyze the noise within a point cloud is to identify reflectance from a smooth (e.g., flat) surface such as pavement or concrete wall, and to check the variability of the point cloud within this surface (e.g., planar) in terms of amplitude and distribution (symmetric, biased, etc.). Such evaluation shall be performed over different distances to provide guidance on sensor usage. In absence of smooth surfaces, or as an alternative, it is also possible to analyze details of repetitive objects such as tile patterns on a roof or railroad tracks. This characteristic is less metric but indicates the smallest detection of detail(s) over certain distance. Such analysis will be performed on different types of surface: roof, road, concrete wall - the later only in the case of mobile, terrestrial mapping.

\subsubsection{Small object detection and vegetation penetration}

Detection of small elements such as wires or penetration of vegetation is one of the main advantages of LiDAR technology 
with respect to passive optical sensing. For this reason, we compare the automotive sensor with the mapping standard also in these aspects.

Detection of wires, thin objects and vegetation penetration is compared in the airborne scenario with the reference LiDAR VQ480 (Riegl). As the nominal point density is equivalent between both sensors, comparison can be done by counting ground points, or sub canopy points over a common area. As PUCK (Velodyne) features only 2 echoes (first and last), chances to detect intermediate vegetation layer with this sensor are principally lower.

\section{RESULTS}

\subsection{Orientation effects}

The first analysis represents the comparison between AIRINS and APX15. At each image projection center, we computed the direct georeferencing parameters (position and attitude) taking into account the calibrated boresight angles. Figure 6 shows attitude errors for APX15 and Navchip SIMU. For roll and pitch, APX15 offers an accuracy of $0.025^{\circ}$ that is conform to the specifications while for heading, the RMS is about $0.11^{\circ}$, so slightly worse than manufacturer specifications $\left(0.08^{\circ}\right)$. The RMS for Navchip SIMU are somewhat similar for roll and pitch while slightly better for heading $\left(0.08^{\circ}\right)$. More details on Navchip IMUs with continuous comparison to AIRINS are reported in Clausen and Skaloud (2020).
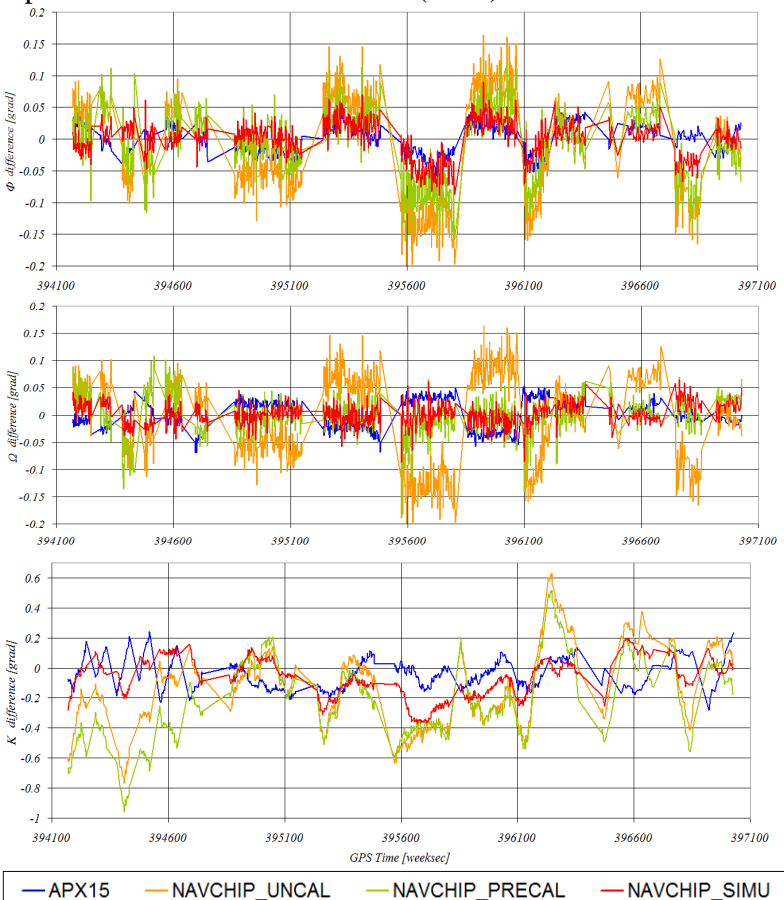

\begin{tabular}{|lll|}
\hline -APX15 -NAVCHIP_UNCAL & -NAVCHIP_PRECAL -NAVCHIP_SIMU \\
\hline Figure 6: Angular difference in [grad] between APX15/Navchip
\end{tabular} and AIRINS for each image projection centre after suppression of boresight. (PHI/pitch (top), OMEGA/ROLL(middle), KAPPA/Heading(bottom)

To express the real and continuous effect of attitude quality on point clouds we generate them using the same high-precision LiDAR with different IMUs: AirINS (as reference), APX15, single Navchip without any pre-calibration, single Navchip with pre-calibration and the SIMU using 4 calibrated Navchips. The comparison of different point clouds is depicted in Figure 7 for a single flight-line as an altimetric difference map (dZ).

On all cases, we can notice that discrepancies spread mostly on the edge of the swath. This is a typical amplification of angular errors, here at AGL > $200 \mathrm{~m}$. For the Navchip, the performance increases significantly using pre-calibration and also the fusion of the 4 IMU's.

Now we investigate two along-track profiles within the point cloud marked by 2 red lines on hillshade in Figure 7 . We notice that $\mathrm{Z}$ error is due to planimetric error at places where terrain is rising (Figure 8 ). This is mostly likely explained by a combined effect of pitch and heading error. Indeed, the heading error in APX15 in a range 0.1-0.2 influences the edge of swath (about $130 \mathrm{~m}$ from the swath center) by a displacement of $30-50 \mathrm{~cm}$. On both cross sections in Figure 8 we can notice for both Navchip and APX15 a planimetric error of about $30-45 \mathrm{~cm}$. Figure 9 tends to suggest that on this strip APX15 gives better results than Navchip-SIMU, but on the cross strips (Figure 8), the synthetic Navchip provides less discrepancies. The lower RMS in heading for this sensor (Sec. 4.1) may explain that.

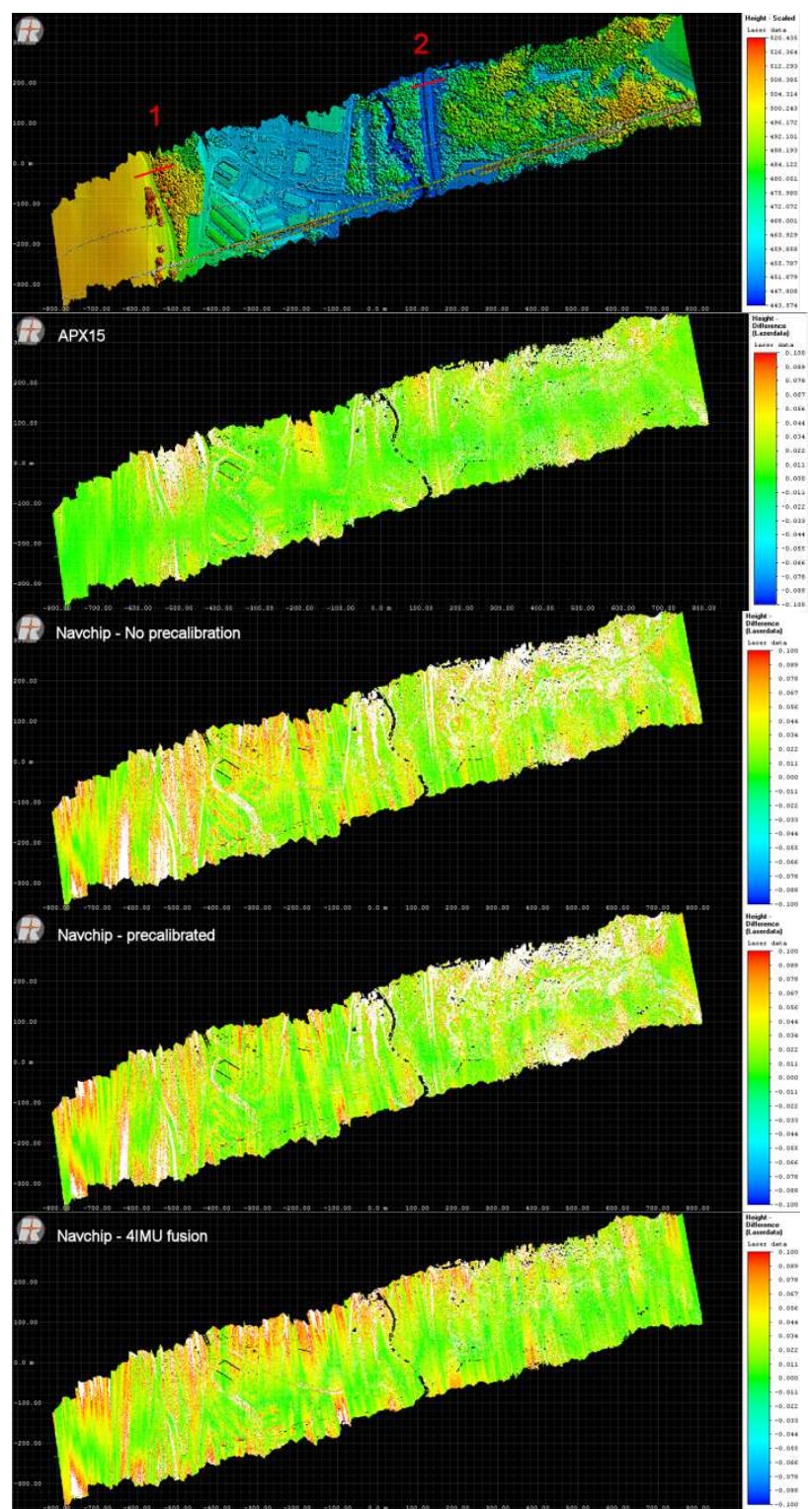

Figure 7: Reference hillshade (top plot) generated with AIRINS and the differences in elevation for point-clouds based on (1) APX15, (2) 1Navchip w.o. precalib., (3) 1Navchip w. precalib., (4) Navchip-SIMU. The scale goes from 0 (green) to $+/-10 \mathrm{~cm}$ (red/blue). White areas are greater than $+/-10 \mathrm{~cm}$. 


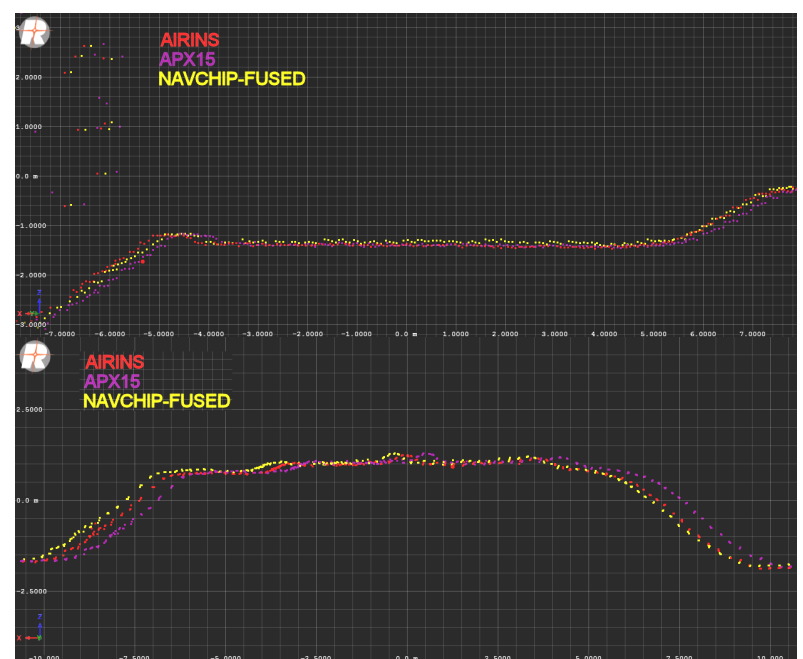

Figure 8: Cross section 1 (top) and 2 (bottom) at edge of swath. Red cloud is AIRINS reference. Planimetric difference is the main error, not detected on flat surface but manifesting immediately with slopes.

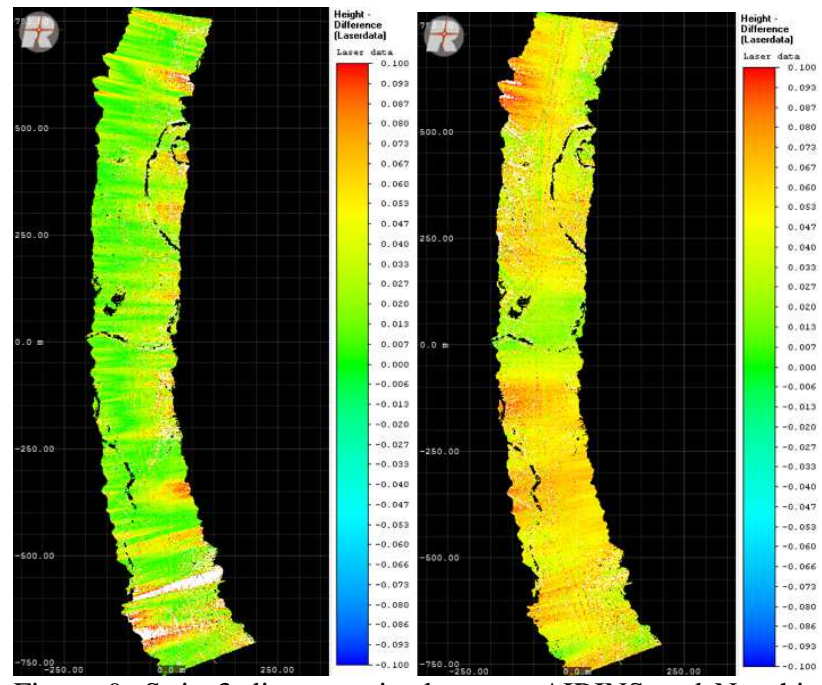

Figure 9: Strip 3 discrepancies between AIRINS and Navchip (fused) on left or APX15 on right.

\subsection{PUCK DTM accuracy and noise}

To estimate DTM accuracy of the automotive LiDAR we have oriented Puck (Velodyne) and VQ480 (Riegl) observations with the same reference IMU (AIRINS). Then we extracted the ground with same extraction parameters in Terrascan (Terrasolid) and controlled the results manually. Absolute $\mathrm{Z}$ error is obtained from GCP's and the relative via subtraction of regular altimetric grids (DTM) based on these point clouds (Figure 10). From this study we can see that:

- The noise distribution of Puck is not symmetric and is centered below the real surface. The systematic component is about $12 \mathrm{~cm}$ and standard deviation is about $20 \mathrm{~cm}$.

- The mean range bias affects the automated process of ground extraction within the Terrascan algorithm as that is based on triangulation of lowest points (after elimination of isolated outliers). Handling this issue would require employing denoising, however, most of available algorithms (Mugner et al., 2019) work on symmetric distributions and therefore do not remove asymmetric noise. This fact explains the observed systematic differences in $\mathrm{Z}$ between both point clouds.

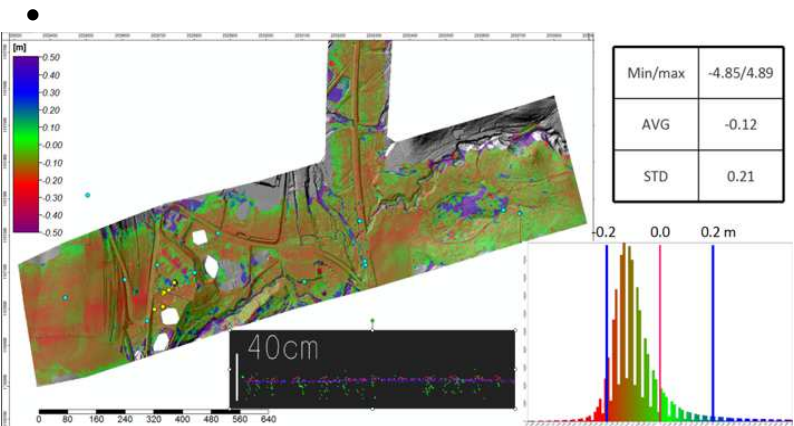

Figure 10: Grid differences between Puck and VQ480 DTM (top left) with a histogram (bottom right) and cross-section (bottom center). On the cross section, red dot is VQ480 and green represents Puck cloud. GCP's are the blue and yellow dots (blue=static GNSS, yellow=RTK GNSS)

The first two rows of Table 4 show the statistics on GCP for both DTM's. The comparison with GCP's confirms the bias and higher noise of Puck-cloud. The $3^{\text {rd }}$ line uses the same analysis for Puck-cloud when generated with APX15. The last line compares the photogrammetric DTM extracted form dense point matching of the RX1RII images. We see that the later provides a comparable performance to the DTM based on VQ480 LiDAR

\begin{tabular}{lccc}
\hline & $\begin{array}{c}\text { Mean dZ } \\
{[\mathrm{m}]}\end{array}$ & $\begin{array}{c}\text { std dZ } \\
{[\mathrm{m}]}\end{array}$ & $\begin{array}{c}\text { Min/max dZ } \\
{[\mathrm{m}]}\end{array}$ \\
\hline \hline VQ480-AIRINS & 0.005 & 0.031 & $-0.065 / 0.054$ \\
Puck-AIRINS & -0.103 & 0.052 & $-0.235 / 0.033$ \\
Puck-APX15 & -0.082 & 0.085 & $-0.265 / 0.063$ \\
Photogrammetry & 0.003 & 0.034 & $-0.082 / 0.074$ \\
RX1RII & &
\end{tabular}

Table 4: Altimetric statistics for DTM error related to GCP's (40)

To deepen the analysis of the noise, we look at local variability on planar surfaces such as road/pavement, which is depicted in Figure 11. There the calculated standard deviation (1 sigma) of Puck is $4 \mathrm{~cm}$ while VQ480 and photogrammetric point cloud are both about $1 \mathrm{~cm}$.

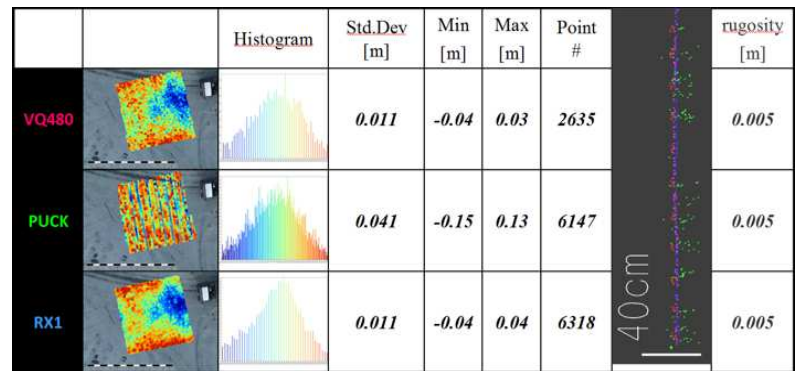

Figure 11: Local variability on a planar surface for different point clouds represented by standard deviation.

Second column in Figure 11 displays the micro relief of a pavement, which is visible on VQ480 and Photogrammetry cloud but not on Puck cloud. The analysis of a micro relief is indeed relevant for detecting pavement deformation (ruts, pothole) or rail tracks. The comparison on a flat, corrugated roof in Figure 11 ( $2^{\text {nd }}$ column) shows the loss of detail with the puck due to the noise. Same figure presents also the variability analysis for this surface. The photogrammetric and VQ480 
point clouds render the details of the roof while only noise is visible on the cloud generated with Puck observations.

\begin{tabular}{|c|c|c|c|c|c|c|}
\hline & Histogram & $\begin{array}{c}\text { Std. Dev. } \\
{[\mathrm{m}]}\end{array}$ & $\begin{array}{c}\mathrm{Min} \\
{[\mathrm{m}]} \\
\end{array}$ & $\begin{array}{c}\text { Max } \\
{[\mathrm{m}]}\end{array}$ & Point \# & $\begin{array}{c}\text { Rugosity } \\
\text { [m] }\end{array}$ \\
\hline va480 & & 0.015 & -0.05 & 0.10 & 1607 & 0.04 \\
\hline PUCK & & 0.055 & -0.17 & 0.36 & 3615 & 0.04 \\
\hline $\mathrm{RX} 1$ & & 0.014 & -0.05 & 0.06 & 2688 & 0.04 \\
\hline
\end{tabular}

Figure 12: Visible level of detail on a corrugated roof. VQ480 and photogrammetry provide a good level of detail of the roof detail.

A cross section on a railroad tracks depicted in Figure 13 indicates the insufficient details for Puck to detect rails. On the other hand, photogrammetry renders the rail, however, in smoother way than VQ480.

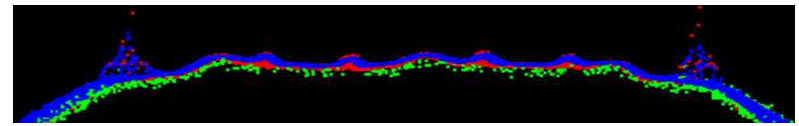

Figure 13: cross section on railroad. Red=VQ480, Green=Puck, Blue $=$ Photogrammetry.

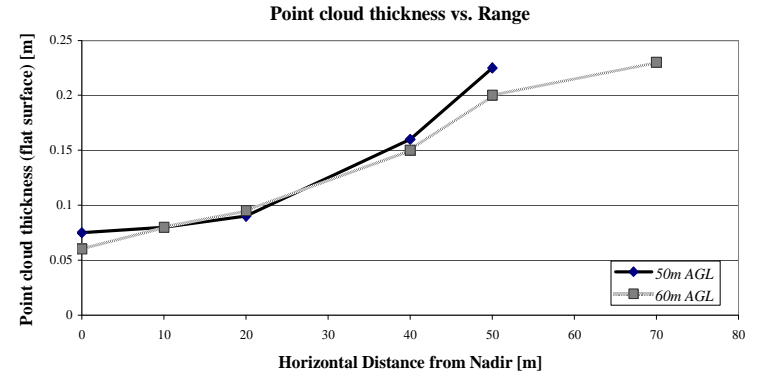

Figure 14: Point cloud thickness (noise) on a flat surface as a function of horizontal distance from nadir at two flying heights.

Lastly, we analyzed the level of noise in Puck point cloud as a function of the range. The manufacture specification states the noise level about 3-4 cm for ranges shorter than $30 \mathrm{~m}$. As flying height below $30 \mathrm{~m}$ are neither safe not practical, we took a flight-line flown at 50 and $60 \mathrm{~m}$ AGL and measured the point cloud thickness at nadir direction. The results are shown in Figure 14. As horizontal distance from nadir increases, the range gets longer with a tangent of beam angle (to nadir) while the incidence angle of the beam decreases. The footprint of laser beam augments with the distance and inversely with the incidence angle on surface. This affects the level of noise that increases 4 to 5 times towards swath extreme compare to nadir direction.

\subsection{Vegetation penetration}

The main advantage of LiDAR over photogrammetry is its capability to measure under canopy. It includes the ground but also intermediate layers of the canopy. This aptitude is shown in Table 5 for 3 forest areas composed of deciduous (zone 1), deciduous and conifer (zone 2) and conifer (zone 3). For each area, we have computed the density of ground points. Puck penetrates canopy efficiently in deciduous area, but its penetration is poor in conifer area. VQ480 provides $10 \%$ more returns from ground that puck despite having lower nominal sampling density. It is partly due to the fact that at $230 \mathrm{~m}$ AGL the VQ480 is far from its range limit whereas Puck at $50 \mathrm{~m}$ AGL is at the limit of its ranging capacity, hence the returns penetrating the canopy are likely too weak to be detected.

Figure 15 illustrates on a cross sections the rendering of point cloud canopy and penetration to the ground for both systems. In deciduous area, Puck signal penetrates to the ground, but the resulting rendering of canopy is poor as the returns are mostly from trunks and large branches. This situation is opposite in conifer area: few returns from ground and well reconstructed canopy. This tends to confirm that the detected reflections of Puck come only from large surfaces.

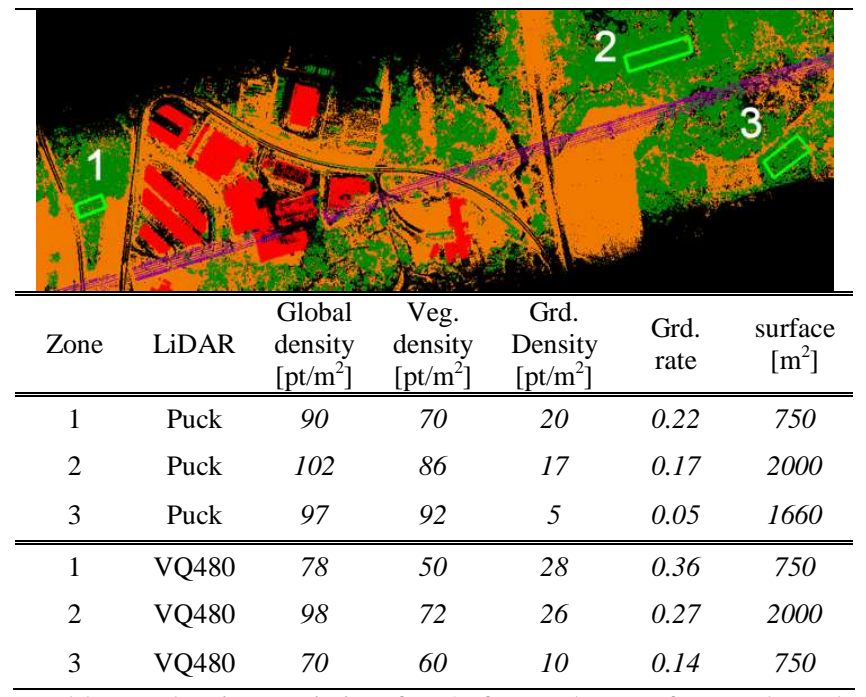

Table 5: density statistics for 3 forested area for Puck and VQ480 LiDAR.

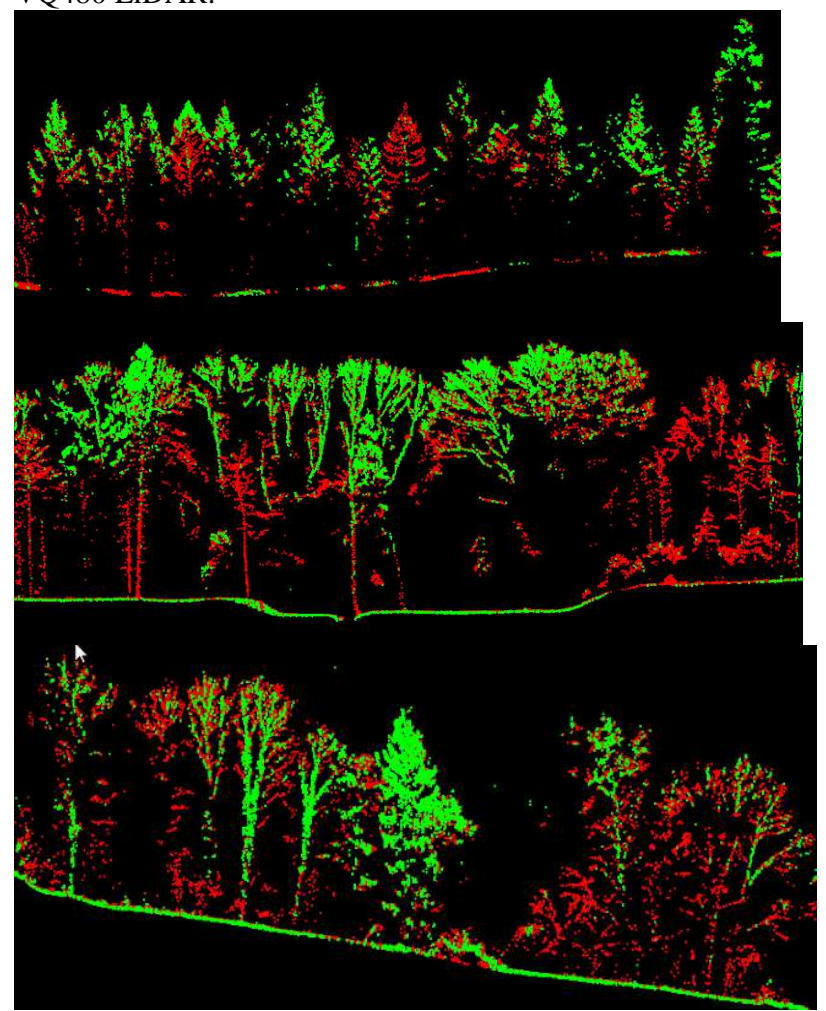

Figure 15: $1 \mathrm{~m}$ width cross section in canopy for zone 3 (up), 2 (middle) and 1(bottom). Green=Puck, Red=VQ480. 


\subsection{Mobile mapping analysis}

As the Puck LiDAR shows a noisy point cloud in airborne application, we analyze closer its ranging capability in terrestrial mobile mapping. Our analysis relies on comparison at different acquisition and return modes: vehicle speed at $18 \mathrm{~km} / \mathrm{h}$ and $36 \mathrm{~km} / \mathrm{h}$ at scanning frequencies $10-20 \mathrm{~Hz}$ and Strongest, Last or Dual, respectively.

We also evaluate its noise level on planar surfaces as a function of range. Table 6 shows the noise ( 1 sigma) on 17 flat surfaces, distributed over $4 \mathrm{~m}$ and $15 \mathrm{~m}$ distance, for each vehicle speed, scan rate and return mode. Vehicle speed and higher scan rate increases (slightly) the noise, but the choice of return mode does not seem to affect it.

\begin{tabular}{cccc|ccc}
\hline \multirow{2}{*}{$\begin{array}{c}\text { Scan } \\
\text { Frequency } \\
{[\mathrm{Hz}]}\end{array}$} & \multicolumn{5}{c}{18} & \multicolumn{4}{c}{ Vehicle speed $[\mathrm{km} / \mathrm{h}]$} \\
\cline { 2 - 7 } & \multicolumn{5}{c}{ Noise $(1 \sigma)[\mathrm{cm}]$ vs Scan Mode } \\
\hline \hline 5 & Dual & Last & Strongest & Dual & Last & Strongest \\
\hline \hline 10 & $\mathbf{2 . 0 2}$ & 1.88 & 2.01 & $\mathbf{2 . 1 3}$ & 2.02 & 1.93 \\
20 & 2.00 & 1.70 & $\mathbf{2 . 3 2}$ & $\mathbf{2 . 2 7}$ & 1.98 \\
\hline $\mathbf{2 . 0 4}$ & 2.02 & 2.01 & 2.06 & $\mathbf{2 . 2 6}$ & $\mathbf{2 . 4 5}$ \\
\hline
\end{tabular}

Table 6: Noise in $\mathrm{cm}$ on Nadir range at $4 \mathrm{~m}$ vs. scan mode, frequency and vehicle speed.

To estimate the absolute accuracy of the Puck point cloud, we compared it with high-end system - VUX1 scanner from Riegl. Point density is 3-6 times higher with dual VUX1 than with single Puck so rendering detail is obviously different between both systems. We have compared point clouds on a short tunnel and on buildings walls using cloud2could routine in Cloud Compare software. The differences in a "tunnel shape" are illustrated in Figure 16 and 17. Thanks to good GNSS visibility before/after underpass, the position error does not affect the point cloud, but error in Puck-cloud orientation is observed and is due to lower angular accuracy of APX15 as compared to navigation grade IMU from IGI. This is confirmed by the fact that larger discrepancies are observed at longer distances, i.e., on the walls opposite to trajectory lane (Figure 18). In short distances, the noise of Puck is only 30 to $50 \%$ bigger than the VUX1.
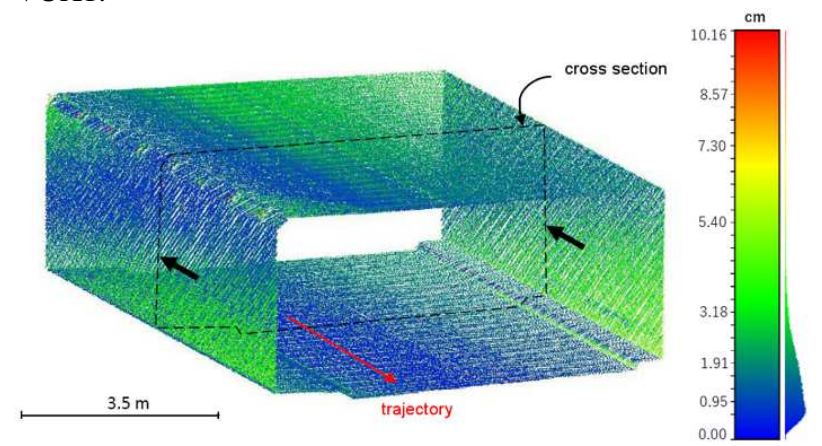

Figure 16: Differences between Puck-VUX point clouds in a tunnel.

The rising of range and reduction of beam incidence angle increase the difference with VUX1. This is mainly due to beam divergence that is 11 times bigger on Puck. We notice that average difference on the perpendicular wall is $14 \mathrm{~cm}$ while the one on parallel facade is $6.7 \mathrm{~cm}$.

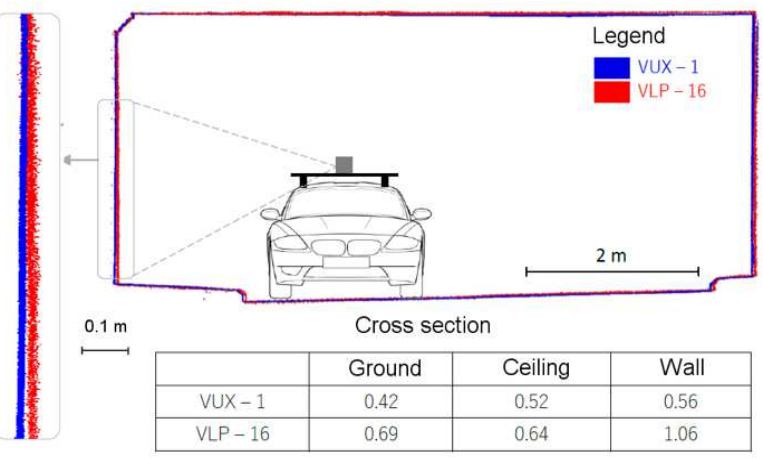

Figure 17: Difference between VUX1 and PUCK point cloud on a tunnel section. The histogram on fig. 16 the distribution of differences according to surface normal while the table at bottom shows the surface noise for each scanner.
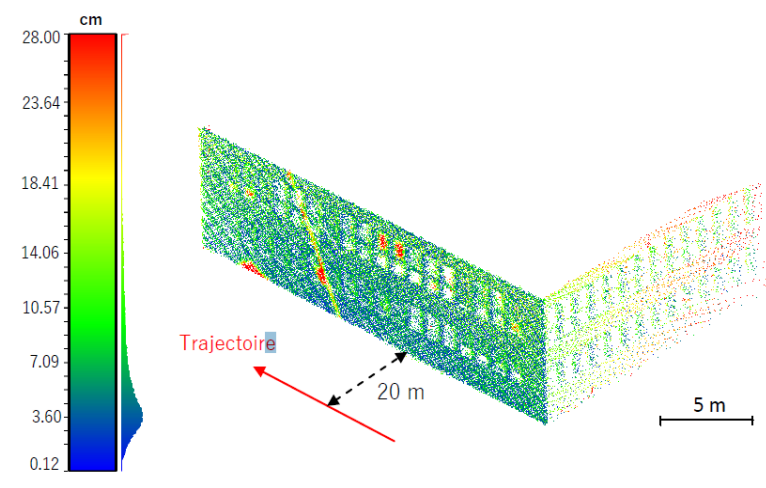

Figure 18: Difference VUX1-PUCK on a building. Rising of range increases the difference.

\section{PRODUCTIVITY ANALYSIS}

Many drone manufacturers advertise their product as apt for "high productivity mapping" thanks to automation and lower cost in data acquisition. However, it is not clear from which size the UAV approach becomes less economical compared to classical airborne acquisition. To which extent is UAV mapping suitable for special corridor application such as power lines or railroads? As a service provider using both, manned helicopters and UAVs, we have analyzed the costs for each type of carrier to estimate the intersecting conditions. We based our cost estimation on the following assumptions:

- Respecting EU flight regulation for UAV: maximum takeoff weight (TOW) $25 \mathrm{~kg}$, max AGL $150 \mathrm{~m}$ and flying within the visual line of sight (VLOS), which is at a radius $\sim 1 \mathrm{~km}, 2$ operators in both cases

- $\quad$ Use of VRS or local base for RTK/PPK GNSS

- Equipment amortization in 100 uses over 3 years

- Corridor width of $100-200 \mathrm{~m}$ which is typical for power line or railroad.

- $\quad$ Flying autonomy of a multi-rotor UAV of 20-25 min

- Final product is DTM/DSM/Orthophoto

- $\quad$ Flight parameters for corridor mapping as in Table 7

\begin{tabular}{lcccc}
\hline & $\begin{array}{c}\text { Coverage } \\
\text { per } 1 \mathrm{~h}\end{array}$ & \# img /km & \# strip & Height AGL \\
\hline \hline UAV-Puck & $4 \mathrm{~km}$ & 250 & $2-3$ & $50 \mathrm{~m}$ \\
Helicopter & $40 \mathrm{~km}$ & 15 & 1 & $270 \mathrm{~m}$ \\
\hline
\end{tabular}

Table 7: Flight parameters for corridor of $200 \mathrm{~m}$ width 
Corridor 200m, Density >40pt/m2, DTM-DSM-Ortho $5 \mathrm{~cm}$

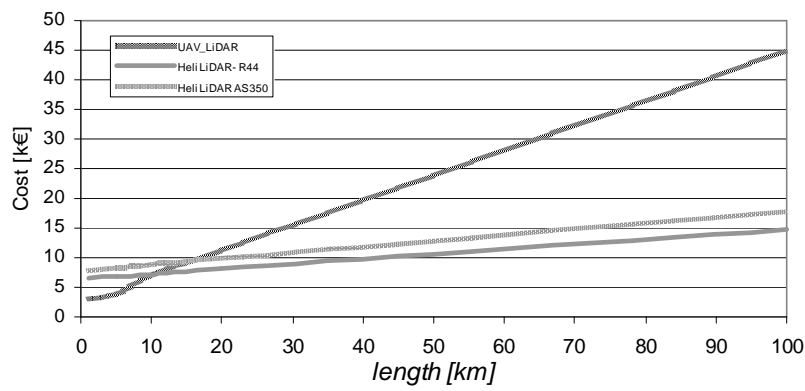

Figure 19: Cost curve for manned and unmanned LiDAR on corridor mapping.

We have taken into account the non-linear scale of processing costs but as we are not looking into large volume, the approximation is close to reality. Figure 19 shows that below 10 $\mathrm{km}$, a UAV is more cost effective than helicopter in a condition when the terrain is easily accessible (i.e., not in mountains). Above this distance, a small and fuel economic helicopter such Robinson R44 is more efficient. If we replace Puck by a miniVUX (Riegl), we can increase AGL to about $100 \mathrm{~m}$ (instead of $50 \mathrm{~m}$ ) and thus gain about $10-20 \%$ in productivity. There the limitation comes mainly from regulation that obliges to cover section of max $2 \mathrm{~km}$ to maintain VLOS with UAV. As long as this limitation remains, larger ${ }^{4} \mathrm{UAV}$ that can carry scanners will not be efficient for medium coverage. Accessibility to terrain remains also a limiting factor. If the operator takes a chopper to access the area, usage of UAV becomes much more expensive than helicopter-borne scanning!

This simulation can be used for small/medium surface mapping. The market of small surface LiDAR is generally for forested areas as opened areas can be surveyed with UAVphotogrammetry. Market prices on such type of jobs showed that above $300-400$ ha, LiDAR UAV is not economically efficient, although sometimes, fashion remains stronger than efficiency!

\section{CONCLUSIONS}

The study was motivated by very optimistic spotlight advertisements presented by UAV mapping sector. We first designed a practical comparison between photo, LiDAR and inertial UAV sensors with respect to larger airborne equipment. The somewhat unique capability to test in a single flight all sensors was possible through a realization of a special mount holding all sensors on one rigid platform that is carried by a helicopter at altitudes and speeds mimicking UAV flights. This created the same physical input to all sensors and thus the possibility of epoch-to-epoch comparison.

We have shown that the use of MEM's IMU such as Applanix APX15, commonly use in UAV market, conforms to manufacturer specifications in roll and pitch but its performance is slightly worse in heading. It showed also practically the limit of such IMUs when flying with LiDAR at higher altitude. Indeed, to keep the planimetric errors induced by attitude errors (mainly heading and pitch) below $10 \mathrm{~cm}$, the flying height above terrain should be limited to $80 \mathrm{~m}$. The same study showed also an important perspective for low cost redundant IMU's based on synthetic fusion technique that is rivaling

\footnotetext{
${ }^{4}$ Some small fixed-wing UAVs with $\mathrm{TOW}<2 \mathrm{~kg}$ are eligible for BVLOS yet not able to carry sufficiently performant LiDAR.
}

APX15 performance at fractional cost of hardware despite being based on relatively old sensors (manufactured in 2011).

As employment of Velodyne's Puck was a trend by many service providers at the time of this study, we dressed a deeper analysis on its potential and limits for airborne and mobile mapping. In this respect, we were a bit disappointed by its aerial performance, where its actual noise is far beyond the published specifications for distances $>50 \mathrm{~m}$. Indeed, the resulting DTM has a noise at least $5 \mathrm{~cm}(1 \sigma)$. On the practical aspects, the ground extraction from its observations is more time consuming because the asymmetric noise for which the tested denoising techniques were not efficient. Nevertheless, the sensor can be used on small projects for forested areas or for wire / power-line detection at AGL of $30-40 \mathrm{~m}$. In other words, the there is a certain consistency in the price and performance ratio between the two LiDAR instruments for airborne applications ( $\sim 8 \mathrm{k} €$ vs. $120 \mathrm{k} €)$. In this respect employment of a sensor like Riegl MiniVUX on UAVs has lower noise and higher ranging capacity, however, for larger volume, weight and price.

We tested the same Velodyne's Puck LiDAR in terrestrial mobile mapping. At close ranges up to $20 \mathrm{~m}$, its noise remains below manufacturer specifications $(2-3 \mathrm{~cm})$, however, the detection of small deformation remains difficult (e.g., ruts $<1.5$ $\mathrm{cm})$. Nevertheless, globally the sensor provides a clean point cloud that could be used for road GIS mapping. Of course, the orientation performance of APX15 is challenging for land use whenever outages in GNSS signal reception occur. Overall, we conclude that usage of Puck represents a good alternative to low-cost solution for mobile mapping for objects at $<30 \mathrm{~m}$ from the surveying vehicle. A suitable denoising strategy that remained to be found could further improve the quality of this (originally automotive) LiDAR sensor.

In the last part of this contribution we focused on the economic aspects of LiDAR corridor mapping performed by small UAV and manned helicopter. We concluded within current European regulations; UAV LiDAR remains economically attractive for small surveys below 300-400 ha.

\section{ACKNOWLEDGEMENTS}

We would like to thank Laurine Cartier, from INSA-Strasbourg, for the work done on Mobile mapping analysis during her internship at Helimap in 2018.

\section{REFERENCES}

Baltsavias, E., 1999. Airborne laser scanning: Existing systems and firms and other resources, ISPRS Journal of Photogrammetry and Remote Sensing, 54(2-3):164-198.

Colomina, I., Molina, P., 2014. Unmanned aerial systems for photogrammetry and remote sensing: A review. ISPRS Journal of Photogrammetry and Remote Sensing, 92: 79-97.

Clausen, P., Skaloud, J., 2020. On the calibration aspects of MEMS-IMUs used in micro UAVs for sensor orientation. Proceedings of IEEE-ION Position Location and Navigation Symposium (PLANS), Portland, OR, USA.

Glennie, C. L., 2007. Rigorous 3D error analysis of kinematic scanning LIDAR systems, J. of Applied Geodesy, 1:147-157. 
Glennie, C. L., Kusari, A., Facchin, A., 2016. Calibration and stability analysis of the VLP-16 laser scanner, International Archives of the Photogrammetry, Remote Sensing and Spatial Information Sciences. XL-3/W4, 55-60.

Gressin, A., Vallet, J., Bron, M., 2020. About photogrammetric UAV-mapping: which accuracy for which application? The International Archives of the Photogrammetry, Remote Sensing and Spatial Information Sciences, XLIII-B1-2020.

Kluter T., 2012. Gecko4nav Technical reference manual v. 1.0., Bern University of Applied Sciences.

Schenk, T., 2001. Modeling and analyzing systematic errors in airborne laser scanners, Technical Report in Photogrammetry No. 19, Ohio State University.

Skaloud, J., Legat, K., 2006. CAMEO - camera exterior orientation, Software description No. 6.0697, Swiss Federal Institute of Technology Lausanne (EPFL).

Skaloud, J., 2017. LIEO - lidar orientation and point cloud generation, Software description No. 6.0704, Swiss Federal Institute of Technology Lausanne (EPFL).

Mugner, E., Seube, N., 2019. Denoising of point clouds. The International Archives of the Photogrammetry, Remote Sensing and Spatial Information Sciences, Volume XLII-2/W17, 217224. 